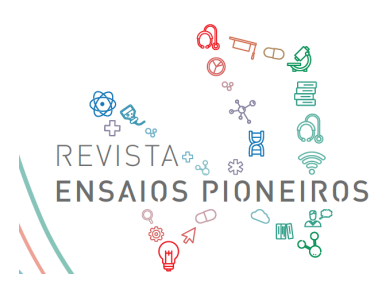

http://ensaiospioneiros.usf.edu.br

\title{
LESÕES INCISIONAIS TRATADAS COM HYPERICUM PERFORATUM L. E ELETROESTIMULAÇÃO
}

INCISIONAL INJURIES TREATED WITH HYPERICUM PERFORATUM L. AND ELECTROSTIMULATION

LEITÃO, Dauciane Pinto da Silva ${ }^{1}$; SANTOS, Luciene Almeida $\operatorname{dos}^{1}$; PAVAN, Joseane ${ }^{1}$; FLORENZIANO, Rosimere Farias de Mendonça ${ }^{2}$; ESQUISATTO, Marcelo Augusto Marretto $^{3}$; ANDRADE, Thiago Antonio Moretti de ${ }^{3}$; SANTOS, Gláucia Maria Tech dos ${ }^{3}$

${ }^{1}$ Graduando do Curso de Biomedicina - Centro Universitário da Fundação Hermínio Ometto FHO; ${ }^{2}$ Mestrando do Programa de Pós-graduação em Ciências Biomédicas Centro Universitário da Fundação Hermínio Ometto - FHO; ${ }^{3}$ Professor do Programa de Pósgraduação em Ciências Biomédicas Centro Universitário da Fundação Hermínio Ometto FHO

marcelosquisatto@fho.edu.br

\begin{abstract}
RESUMO. O presente trabalho estudou efeitos de Hypericum perforatum associado ou não à eletroestimulação na cicatrização da pele de ratos. Trinta e seis animais foram divididos em (n=9): (C) sem tratamento; (MC) tratado com eletroestimulação; (HYP) tratado com $H$. perforatum; (HYP+MC) tratado com H. perforatum+eletroestimulação. Amostras foram coletadas dos animais eutanasiados no $2^{\circ}, 6^{\circ}$ e $10^{\circ}$ dia após a lesão cirúrgica para análise histomorfométrica: quantificação de infiltrado inflamatório, vasos sanguíneos, fibroblastos e porcentagem da área de colágeno. Para análise estatística foi utilizado ANOVA Two-way e pós-teste de Tukey (média \pm erro padrão). Os resultados mostraram que o infiltrado inflamatório foi menor em HYP e MC+HYP em relação a $\mathrm{C}$ e $\mathrm{MC}$ no $2^{\circ}$ e $6^{\circ}$ período experimental; no dia 10 a eletroestimulação promoveu aumento do número dessas células. Em relação aos vasos sanguíneos observou-se que MC apresentou maiores valores no $10^{\circ}$ período experimental em relação aos demais grupos. No $2^{\circ}$ e $6^{\circ}$ dia após a lesão, MC+HYP mostrou maior número de fibroblastos em relação aos demais grupos experimentais, o mesmo ocorrendo no $6^{\circ}$ dia em $\mathrm{MC}$ e HYP em relação a C. Quanto à porcentagem de área de colágeno observou-se aumento em HYP e MC+HYP no $2^{\circ}$ dia em relação a C e MC. Diante disso, pode-se concluir que $H$. perforatum em formulação homeopática neste modelo experimental diminuiu inflamação, promoveu fibroplasia e colagênese. A eletroestimulação diminuiu inflamação e estimulou angiogênese; em associação ou não ao $H$. perforatum promoveu fibroplasia. Portanto, essas terapias podem ser indicadas como alternativas no reparo de lesões incisionais.
\end{abstract}

Palavras-chave: cicatrização, fitoterapia; eletroestimulação.

ABSTRACT. The present study studied the effects of Hypericum perforatum associated or not with electrostimulation on rat skin healing. Thirty-six animals were divided into $(n=9)$ : (C) without treatment; (MC) treated with electrostimulation; (HYP) treated with H. perforatum; $(\mathrm{HYP}+\mathrm{MC})$ treated with $H$. perforatum+electrostimulation. Samples were collected from euthanized animals (2nd, 6th and 10th day after surgical injury) for histomorphometric analysis: quantification of inflammatory infiltrate, blood vessels, fibroblasts, and percentage of collagen area. Statistical analysis was performed using two-way ANOVA (Tukey post-test). The results showed that the inflammatory infiltrate was lower in HYP and MC+HYP compared to $\mathrm{C}$ and $\mathrm{MC}$ in the 2nd and 6th experimental period. On day 10, electrostimulation promoted an increase in the number of these cells. Regarding blood vessels, it was observed that MC presented a 


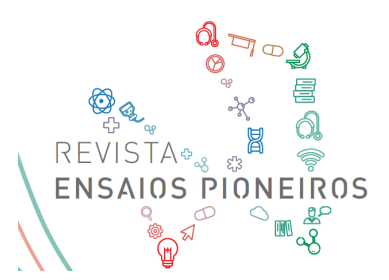

higher values in the 10th experimental period compared to the other groups. On the 2nd and 6th day after the lesion, MC+ YP showed a higher number of fibroblasts than the other experimental groups, the same occurring on the 6th day after MC and HYP compared to C. As for the percentage of collagen area, an increase was observed. in HYP and MC+HYP on the 2nd day compared to $\mathrm{C}$ and $\mathrm{MC}$. Therefore, it can be concluded that $H$. perforatum in the homeopathic formulation in this experimental model decreased inflammation, promoted fibroplasia, and collagenesis. Electrostimulation decreased inflammation and stimulated angiogenesis; whether or not associated with $H$. perforatum promoted fibroplasia. Therefore, these therapies may be indicated as alternatives in the repair of incisional injuries.

Keywords: wound healing, herbal medicine; electrostimulation.

\section{INTRODUÇÃO}

A cicatrização de lesões é um processo importante, porém complexo, sendo governado por fases sequenciais, mas sobrepostas. Essas incluem complexa cascata de eventos bioquímicos e celulares com objetivo de restaurar a integridade e a função dos tecidos envolvidos e denominam-se fases de hemostasia, inflamatória, proliferativa e de remodelação (WANG et al., 2018).

Diferentes modalidades de terapias que acelerem o processo de cicatrização tecidual são de grande interesse para os profissionais da saúde e pesquisadores, apresentando promissores resultados como, por exemplo, a fitoterapia que possui várias indicações terapêuticas (SANTOS et al., 2013; AKBIK et al., 2014; MOURAO et al., 2014; MAVER et al., 2015; JARIć et al., 2018; DAS; BEHERA; PRAMANIK, 2017; POLETTI et al., 2018;). Também a homeopatia tem sido amplamente utilizada (NORRED, 2002; CAMPOS, BENIN, CAMARGO, 2010) sendo que seu principio envolve a aplicação de doses mínimas e pouco invasivas.

Hypericum perforatum L. demonstrou propriedades cicatrizantes no tratamento de lesões (VANDENBOGAERDE e DE WITTE, 1995; SÜNTAR et al., 2010; EGILMEZ; KOKTEN; KALCIOGLU, 2017). Provavelmente sua ação no processo de cicatrização deva-se por seus numerosos constituintes biologicamente ativos, incluindo hypericina e seus derivados, hyperforina, flavonóides, catequinas, epicatechinas, procyanidina B2, amino ácidos derivados e óleos essenciais (RAO et al.,1991). Birt et al. (2009) verificaram que constituintes desta espécie foram capazes de inibir a produção de Prostaglandina E2 em macrófagos, o que sugere grande potencial terapêutico anti-inflamatório. Também foram relatadas propriedades pró e anti-inflamatórias do fitoterápico (EGILMEZ et al. (2017).

Atualmente a microcorrente atua sobre os tecidos em nível celular restaurando a bioeletricidade. Sua aplicação é subsensorial não estando associada à sensação desconfortável como outras correntes elétricas, ou seja, é indolor, além de não apresentar efeitos colaterais, ser de baixo custo e de fácil aplicação (MARTELLI et al., 2016). A utilização da eletroestimulação também tem se mostrado bastante promissora e experimentos com correntes elétricas de baixa intensidade (microcorrente) demonstraram efeitos importantes na cicatrização de diferentes tecidos (MENDONÇA et al., 2009; PASSARINI et al., 2012; MOBINI; LEPPIK; BARKER, 2016; SPADARI et al., 2017; FONSECA et al., 2018). A eletroestimulação induz o fluxo de elétrons na pele e tem sido utilizada para restabelecer a atividade bioelétrica endógena de tecidos danificados. Esse processo é chamado de bioestimulação, pois é compatível com o das correntes endógenas que atuam no organismo no nível celular (KLOT, 2005; BAYAT et al., 2005). Eletroestimulação induz aumento no número de fibroblastos na área lesionada e aumento na produção de colágeno (CASTRO et al., 2012; NEVES et al., 2013). 


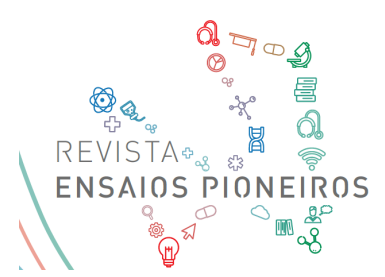

Esta terapia representa um excelente instrumento para promover vascularização durante o processo de cicatrização (MARTELLI et al., 2016). Migliato et al. (2011), através de estudos histomorfométricos observaram com a aplicação da microcorrente de modo contínuo, com intensidade de $10 \mu \mathrm{A}$, frequência de $0,3 \mathrm{~Hz}$, durante 2 minutos em feridas incisionais em ratos Wistar favoreceu o aumento da área de reparo e o número total de células. Após 6 e 10 dias da lesão, a área da ferida foi completamente reepitelizada com a observação da derme preenchida com tecido fibroso, fibras de colágeno reorganizadas e elementos de fibrilas compactados.

O presente trabalho tem como objetivo analisar histomorfometricamente os efeitos da aplicação tópica de $H$. perforatum L. em formulação homeopática associada à eletroestimulação no reparo de lesões incisionais em ratos.

\section{METODOLOGIA}

\section{Modelo Experimental}

Foram utilizados 36 ratos Wistar (Rattus norvegicus) machos, com 90 dias e peso médio de $250 \mathrm{~g}$, obtidos do Centro de Experimentação Animal do Centro Universitário da Fundação Hermínio Ometto - FHO. Todos os procedimentos experimentais e cirúrgicos foram realizados após aprovação pelo Comitê de Ética em Uso Animal - CEUA do Centro Universitário da Fundação Hermínio Ometto - FHO (Parecer nº58/2018).

Os animais foram divididos em quatro grupos, distintos $(\mathrm{n}=$ nove), a saber: $(\mathrm{C})$ Grupo controle - sem tratamento; (MC) Grupo tratado com aplicação de eletroestimulação (10 mA/2 min); (HYP) Grupo tratado com aplicação tópica de gel contendo $H$. perforatum $(3 \mathrm{dH}$ e $10 \%$ ); (HYP+MC) Grupo tratado com aplicação tópica de gel contendo H. perforatum ( $3 \mathrm{dH}$ e $10 \%$ ) + aplicação de eletroestimulação (10 mA/2 min).

\section{Protocolo experimental}

Foi realizada tricotomia na região dorsal de todos os animais após anestesia com associação de cloridrato de ketamina ( $1 \mathrm{ml} / \mathrm{kg}$ peso) e cloridrato de xilazina $(0,2 \mathrm{ml} / \mathrm{kg}$ peso). Em seguida foi realizada incisão cutânea, na qual se utilizou lâmina 15 em bisturi convencional montada em cabo número 3, com profundidade de corte de $2 \mathrm{~mm}$. A incisão mediu $40 \mathrm{~mm}$ e foi previamente demarcada com caneta dermográfica, até a exposição da fáscia muscular dorsal.

Após a intervenção todos os animais receberam analgésico por 72 horas (20 gotas de dipirona sódica diluídas em $250 \mathrm{ml}$ de água por dia) e foram alojados em caixas individuais de policarbonato com xilana, em temperatura constante $\left(23 \pm 2^{\circ} \mathrm{C}\right)$, umidade de $55 \%$, ciclo claro/escuro de $12 \mathrm{~h}$ e água e ração durante todo o protocolo experimental.

\section{Tratamento com H. perforatum}

O gel contendo H. perforatum ( $3 \mathrm{dH}$ e $10 \%$ ) foi obtido da Farmácia Homeopática NOVA ERA em Araras-SP (farmácia para composição, homeopatia e produtos naturais; farmacêuticos responsáveis: Sonia C. Rossi Avelar (CRF 10.703), Elaine Jussi Lopes (CRF 40.208), Debora Milene Correa (CRF 45.077), Ana C. L. Basquera (CRF 63.931). O gel consistia em 10\% de Natrosol, $0,2 \%$ de Nipagin, $5 \%$ de Propileno glicol e água qsp. Para a aplicação tópica nas incisões na pele dos ratos utilizou-se espátula com cerca de $20 \mathrm{mg}$ da formulação.

\section{Aplicação de eletroestimulação}




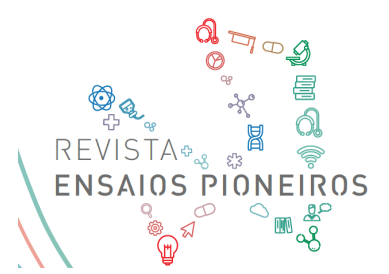

Para aplicação da eletroestimulação (microcorrente) foi utilizado estimulador elétrico transcutâneo (Physiotonus Microcurrent, Bioset, Rio Claro, São Paulo, Brasil). O dispositivo foi configurado para modo de corrente microgalvânica contínua com intensidade de $10 \mathrm{~mA} / 2$ min, de acordo com o protocolo de Mendonça et al. (2009), sendo que dois eletrodos de metal de uma ponta esférica ( $10 \mathrm{~mm}$ de diâmetro) foram colocados ao redor da lesão em quatro pontos cruzados. Não houve necessidade de anestesiar ou conter os animais para o tratamento, pois a corrente elétrica de estimulação é fisiológica, ou seja, não invasiva e não causa desconforto algum. Os animais foram tratados dentro das caixas onde ficaram alojados e os tratamentos iniciados logo após a intervenção cirúrgica e ocorreram diariamente por 10 dias, no mesmo horário e da mesma maneira.

\section{Eutanásia e coleta das amostras}

Três animais de cada grupo foram eutanasiados com aprofundamento anestésico e, posterior, deslocamento cervical, no $2^{\circ}, 6^{\circ}$ e $10^{\circ}$ dia após o procedimento experimental, para a coleta das amostras da área de lesão. Para este propósito, foi removida aproximadamente 60 $\mathrm{mm} / 30 \mathrm{~mm}$ da área da lesão que foi submetida a análise histomorfométrica.

\section{Análise Histomorfométrica}

Após a remoção, os fragmentos do tecido foram imersos em solução fixadora contendo formaldeído a 10\% em tampão Millonig pH 7,4 durante 24 horas, à temperatura ambiente. Em seguida, as peças foram lavadas em tampão e submetidas aos procedimentos padrões para embebição em Paraplast (Histosec ${ }^{\circledR}-$ Merck). Cortes longitudinais das peças com 5,0 $\mu \mathrm{m}$ de espessura foram corados com: Hematoxilina e Eosina (HE) para quantificação do número de infiltrado inflamatório, fibroblastos e vasos sanguíneos, além de Tricômio de Gomori, para quantificação da porcentagem de colágeno. As imagens das secções foram capturadas no Microscópio Leica ${ }^{\circledR}$ DM2000, do Laboratório de Micromorfologia do Centro Universitário da Fundação Hermínio Ometto - FHO. De cada três animais de cada grupo foram capturadas 6 imagens da área da lesão no $2^{\circ}, 6^{\circ}$ e $10^{\circ}$ dia após a lesão, as quais foram analisadas utilizando o software ImageJ. Na quantificação de infiltrado inflamatório, fibroblastos e vasos sanguíneos utilizou-se aumento de 400x e para quantificação e porcentagem da área de colágeno aumento de 200x (ANDRADE et al., 2011). As contagens foram realizadas em duplo-cego, sendo consideradas as médias das contagens das seis imagens/animal.

\section{Análises estatísticas}

Os resultados foram digitados no GraphPad Prism 6 e comparados pelo teste ANOVA Two-Way e pós-teste de Tukey $(\mathrm{p}<0,05)$.

\section{RESULTADOS E DISCUSSÃO}

\section{H. perforatum modula a inflamação e a eletroestimulação promove angiogênese}

Observou-se que o número de infiltrado inflamatório foi menor em $\mathrm{MC}, \mathrm{HYP}$ e $\mathrm{MC}+\mathrm{HYP}$ em relação a $\mathrm{C}$ em todos os períodos experimentais (Figura $1 \mathrm{~A}$ e B). Em relação 
aos vasos sanguíneos observou-se que o grupo tratado com MC apresentou valores maiores em relação aos demais grupos em todos os períodos experimentais (Figura 1C).

A eletroestimulação é capaz de estimular o recrutamento de células inflamatórias para o local da lesão a fim de auxiliar no processo de cicatrização (MENDONÇA, et al., 2009; TANGERINO FILHO et al., 2016; YU et al., 2019), o que foi observado em nossos resultados que corrobora com outros estudos. Células inflamatórias realizam multitarefas no local lesionado, facilitando o desbridamento da lesão e produzindo quimiocinas, metabólitos e fatores de crescimento (EMING; WYNN; MARTIN, 2017). Os tratamentos utilizados neste modelo experimental promoveram diminuição da inflamação. A literatura relata a eletroestimulação apresentando importante ação anti-inflamatória (NAIR, 2018). H. perforatum, em formulação homeopática ou não, promoveu ação anti-inflamatória (CASTRO et al., 2012; ALONSOCASTRO; JUÁREZ-VÁZQUEZ; CAMPOS-XOLALPA, 2016; EGILMEZ et al., 2017; HAMMER et al., 2017).

A eletroestimulação também se apresenta eficaz na formação de novos vasos sanguíneos (angiogênese), o que foi observado neste estudo, sendo de suma importância para a cicatrização de modo a nutrir tecido lesionado (MENDONÇA, et al., 2009; PASSARINI et al., 2012; CASTRO et al., 2012; YU; HU; PENG, 2014).

\section{Eletroestimulação e H. perforatum promovem fibrogênese}

Observou-se que no $2^{\circ}$ e $6^{\circ}$ dia após a lesão $\mathrm{MC}+\mathrm{HYP}$ mostrou maior número de fibroblastos em relação aos demais grupos experimentais. No $6^{\circ}$ dia, além de $\mathrm{MC}+\mathrm{HYP}, \mathrm{MC}$ e HYP também apresentaram maior número de fibroblastos em relação a C. Não se observou diferença entre os tratamentos no $10^{\circ}$ dia experimental (Figura 2 A e B).

\section{H. perforatum promove colagênese}

Quanto à porcentagem de área de colágeno observou-se aumento em HYP e MC+HYP no $2^{\circ}$ dia em relação a $\mathrm{C}$ e MC. Não ocorreu diferença entre os tratamentos no dia 6 e 10 após a lesão (Figura $2 \mathrm{C}$ ).

Ambos os tratamentos utilizados neste estudo, isolados ou associados, promoveram aumento de fibroblastos na região da lesão. Durante a reepitelização, a proliferação celular é um evento essencial, de modo que fibroblastos garantem suprimento adequado de células que migrarão e cobrirão a superfície da lesão (LI, CHEN, KISNER, 2007). Desta forma H. perforatum e eletroestimulação contribuíram satisfatoriamente para a reorganização estrutural da matriz extracelular neste modelo experimental corroborando com dados na literatura (VANDENBOGAERDE; DE WITTE, 1995; SÜNTAR et al., 2010; EGILMEZ; KOKTEN; KALCIOGLU, 2017; THULABANDU; CHEN; ATIT, 2017).

A fibroplasia começa com a formação de tecido de granulação, caracterizada pela proliferação de fibroblastos, principais agentes responsáveis pela deposição da nova matriz (MEDRADO et al., 2010). O principal componente de uma cicatriz do tecido conjuntivo maduro é o colágeno. Os fibroblastos, produtores de colágeno, são recrutados da derme da borda da lesão para sintetizar essa proteína. Durante esse estágio inicial de reparo, o colágeno tipo III é predominante, sintetizado por fibroblastos no tecido de granulação (ISAAC et al., 2010). Com o fechamento da lesão, o colágeno tipo III sofre degradação e a síntese do colágeno tipo I aumenta (GONZALEZ et al., 2016; UEMURA et al., 2016). A porcentagem de área de colágeno mostrou-se aumentada com a utilização de $\mathrm{H}$. perforatum e este associado à eletroestimulação logo no primeiro período experimental estudado, demonstrando que este 
fitoterápico contribuiu satisfatoriamente para deposição de nova matriz extracelular nas lesões incisionais.
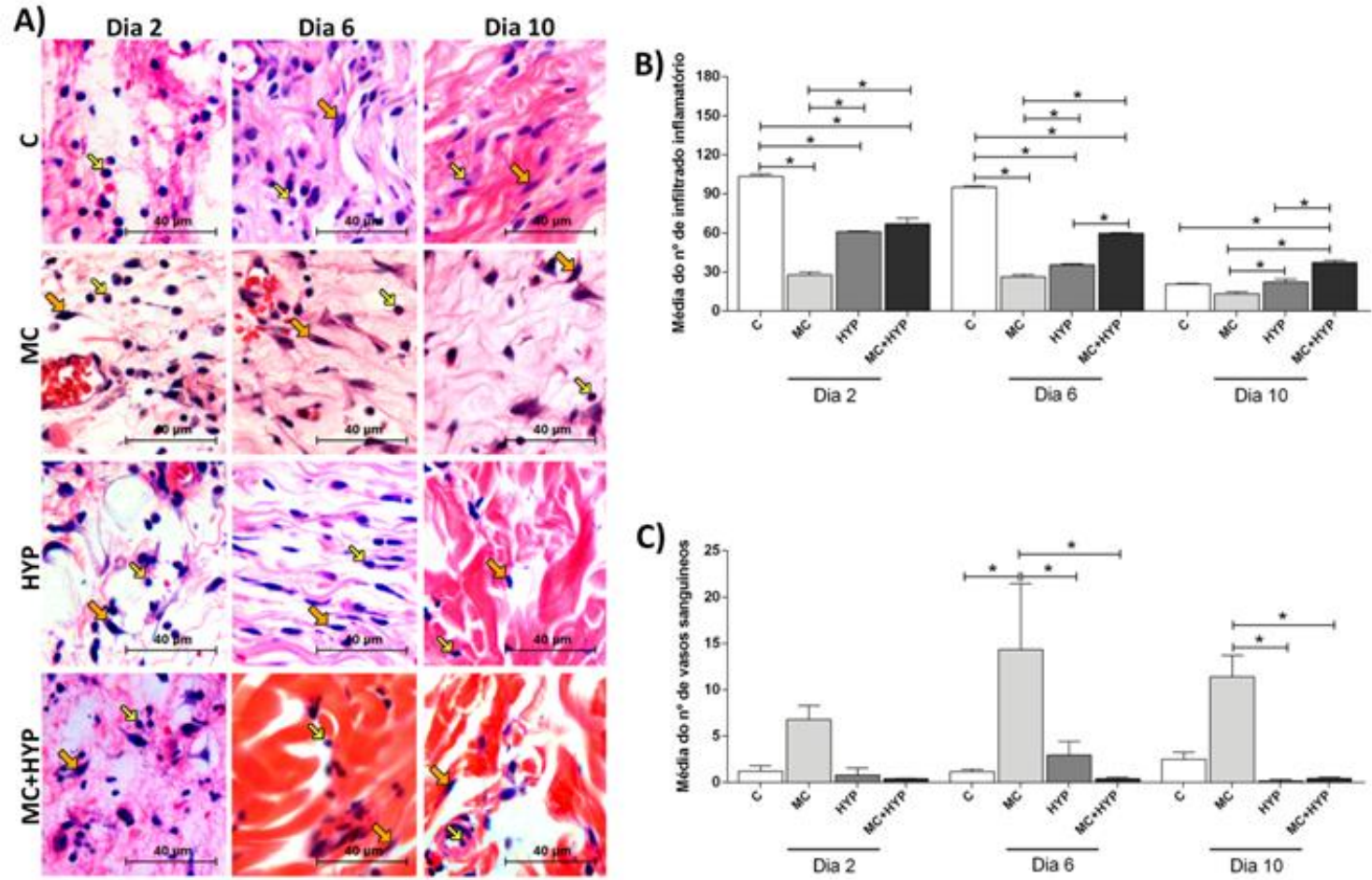

Figura 1: Fotomicrografia representativa de infiltrado inflamatório (setas amarelas) e fibroblastos (setas laranjas) (coloração HE, aumento 400x) (A). Número de infiltrado inflamatório (B) e vasos sanguíneos e (C) dos grupos C, $\mathrm{MC}, \mathrm{HYP}$ e MC+HYP no $2^{\circ}, 6^{\circ}$ e $10^{\circ}$ dia após a lesão experimental. As barras representam a média $\pm \mathrm{EPM}, \mathrm{n}=3$, $\mathrm{p}<0,05$. ANOVA Two-Way e pós-teste de Tukey. 


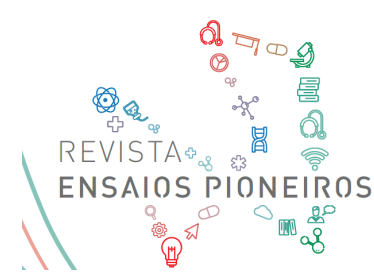

http://ensaiospioneiros.usf.edu.br

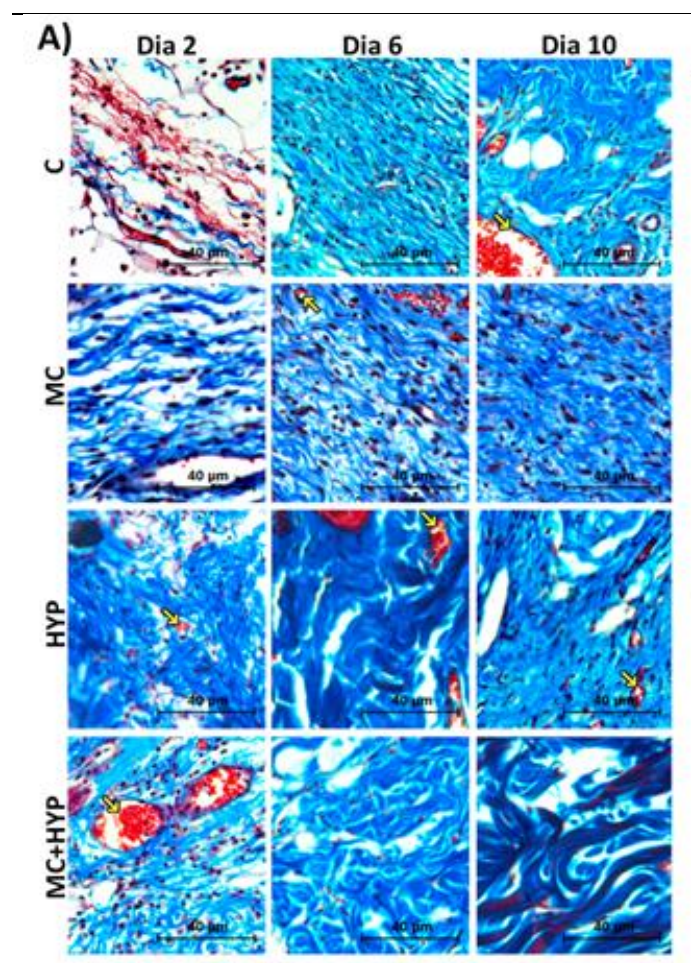

B)
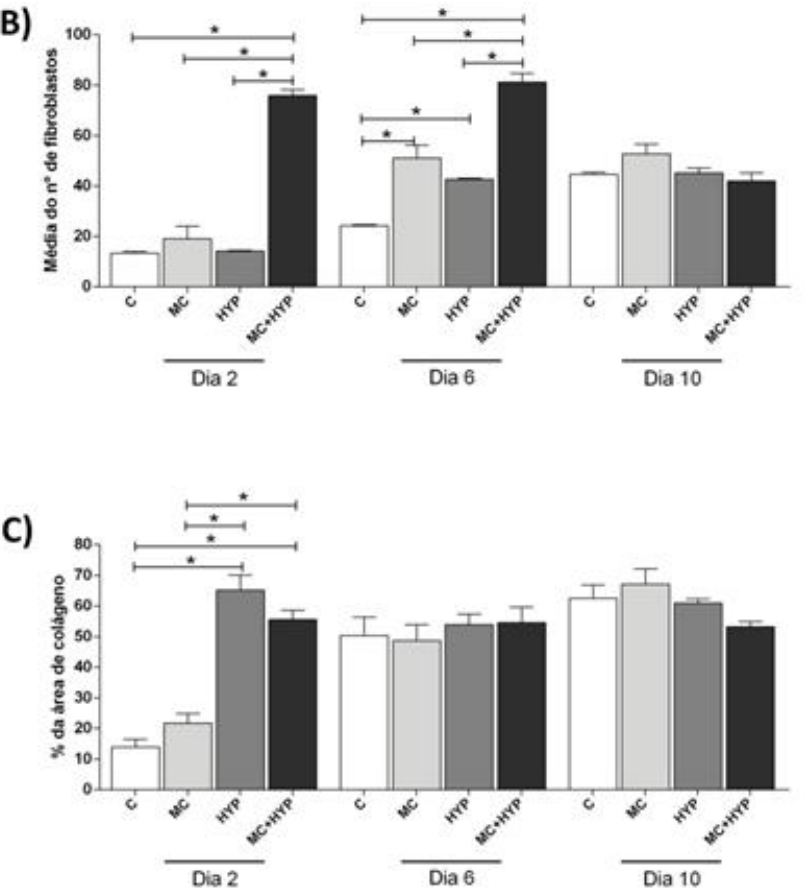

Figura 2: (A) Fotomicrografia representativa da área de colágeno (coloração azul - tricrômio de Gomori, aumento 200x), destacando também os vasos sanguíneos (setas amarelas). Número de fibroblastos (B) e porcentagem de colágeno (C) dos grupos C, MC, HYP e MC+HYP no $2^{\circ}, 6^{\circ}$ e $10^{\circ}$ dia após a lesão experimental. As barras representam a média \pm EPM, $n=3, p<0,05$. ANOVA Two-Way e pós-teste de Tukey.

\section{CONCLUSÃO}

Conclui-se que, neste modelo experimental, $H$. perforatum em formulação homeopática diminuiu a inflamação, promoveu fibroplasia e colagênese nas fases iniciais da cicatrização. A eletroestimulação quando utilizada isoladamente diminuiu a inflamação e estimulou a angiogênese; em associação ou não ao $H$. perforatum promoveu a fibroplasia. Portanto, essas terapias podem ser indicadas como terapias alternativas no reparo de lesões incisionais.

\section{AGRADECIMENTOS}

Os autores agradecem à Fundação Hermínio Ometto pelo suporte financeiro.

\section{REFERÊNCIAS}

AKBIK, D. et al. Curcumin as a wound healing agent. Life Sciences, v. 116, n. 1, p. 1-7, 2014.

ALONSO-CASTRO, A. J.; JUÁREZ-VÁZQUEZ, M. C.; CAMPOS-XOLALPA, N. Medicinal Plants from Mexico, Central America, and the Caribbean Used as Immunostimulants. Evidence-based Complementary and Alternative Medicine, v. 2016, p. 1-15, 2016.

AMADEU, T. et al. Vascularization Pattern in Hypertrophic Scars and Keloids: A Stereological Analysis. Pathology - Research and Practice, v. 199, n. 7, p. 469-473, 2003. 


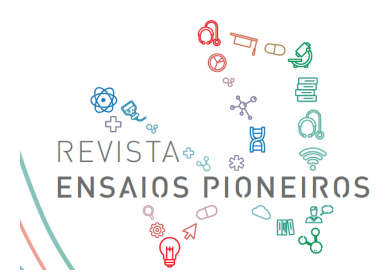

BAYAT, M. et al. Experimental wound healing using microamperage electrical stimulation in rabbits. The Journal of Rehabilitation Research and Development, v. 43, n. 2, p. 219-225, 2006.

BIRT, D. F. et al. Hypericumin infection: Identification of anti-viral and anti-inflammatory constituents. Pharmaceutical Biology, v. 47, n. 8, p.774-782, 2009.

CAMPOS F. L.; BENIN L. A.; CAMARGO V. M. F. Homeopathic approach profile of Veterinary Teaching Clinic (CEVET) at Universidade Estadual do Centro-Oeste (UNICENTRO), Parana, Brazil. Ambiência, v. 6, n. 2, p. 289 - 296, 2010.

CASTRO, F. C. et al. Effects of microcurrent application alone or in combination with topical Hypericum perforatum L. and Arnica montana L. on surgically induced wound healing in Wistar rats. Homeopathy, v. 101, n. 3, p. 147-153, 2012.

DAS, U; BEHERA, S. S; PRAMANIK, K. Ethno-Herbal-Medico in Wound Repair: An Incisive Review. Phytotherapy Research, v. 31, n. 4, p. 579-590, 2017.

DIAO J. S.; XIA, W. S.; GUO S. Z. Bevacizumab: A potential agent for prevention and treatment of hypertrophic scar. Burns, v. 36, n. 7, p. 1136-1137, 2010.

DIPIETRO, L. A. Angiogenesis and wound repair: when enough is enough. Journal of Leukocyte Biology, v. 100, n. 5, p. 979-984, 2016.

EGILMEX, S. A.; WYNN, T. A.; MARTIN, P. Inflammation and metabolism in tissue repair and regeneration. Science, v. 356, n. 6342, p.1026-1030, 2017.

FONSECA, J.H. et al. Electrical stimulation: Complementary therapy to improve the performance of grafts in bone defects. Journal of Biomedical Materials Research Part B: Applied Biomaterials, v. 107, n. 4, p. 924-932, 2018.

GIRA, A. K. et al. Keloids demonstrate high-level epidermal expression of vascular endothelial growth factor. Journal of the American Academy of Dermatology, v. 50, n. 6, p.850-853, 2004.

GONZALEZ, A. C. O. et al. Wound healing - A literature review. Anais Brasileiros de Dermatologia, v. 91, n. 5, p.614-620, 2016.

HULABANDU, V.; CHEN, D.; ATIT, R. P. Dermal fibroblast in cutaneous development and healing. Wiley Interdisciplinary Reviews: Developmental Biology, v. 7, n. 2, p. 307-320, 2017.

ISAAC, C. et al. Processo de cura das feridas: cicatrização fisiológica. Revista de Medicina, v. 89, n. 3/4, p. 1-125, 2010.

JARIC, S. et al. Traditional wound-healing plants used in the Balkan region (Southeast Europe). Journal of Ethnopharmacology, v. 211, p. 311-328, 2018. 
KLOTH, L.C. Electrical Stimulation for Wound Healing: A Review of Evidence From In Vitro Studies, Animal Experiments, and Clinical Trials. The International Journal of Lower Extremity Wounds, v. 4, n. 1, p. 23-44, 2005.

LI, J.; CHEN, J. KIRSNER, R. Pathophysiology of acute wound healing. Clinics in Dermatology, v.54, n. 7, p. 740-751, 2015.

MARTELLI, A. et al. Microcorrente no processo de cicatrização: revisão da literatura. Archives of Health Investigation, v. 5, n. 3, p. 327-345, 2016.

MAVER, T. et al. A review of herbal medicines in wound healing. International Journal of Dermatology, v. 54, n. 7, p. 740-751, 2015.

MEDRADO, A. et al. Phenotype characterization of the pericytes during tissue repair following low- level laser therapy. Photodermatology, Photoimumunology \& Photomedicine, v. 26, n. 4, p. 192-197, 2010.

MENDONÇA, F. A. S. et al. Effects of the application of Aloe vera (L.) and microcurrent on the healing of wounds surgically induced in Wistar rats. Acta Cirúrgica Brasileira, v. 24, n. 2, p.150-155, 2009.

MIGLIATO K.F. et al. Effect of Glycolic Extract of Dillenia indica L. combined with Microcurrent Stimulation on Experimental Lesions in Wistar Rats. Wounds, v. 23 n. 5, p. 11$20,2011$.

MOBINI, S; LEPPIK, L; BARKER, J.H. Direct current electrical stimulation chamber for treating cells in vitro. Biotechniques, v. 60, n. 2, p. 93-98, 2016.

MOURÃO, V. B. et al. Anti-hemorrhagic effect of hydro-alcoholic extract of the leaves of Mikania glomerata in lesions induced by Bothrops jararaca venom in rats. Acta Cirurgica Brasileira, v. 29, n. 1, p. 30-37, 2014.

NAIR, H. K. R. Microcurrent as an adjunct therapy to accelerate chronic wound healing and reduce patient pain. Journal of Wound Care, v. 27, n. 5, p. 296-306, 2018.

NEVES, L. M. G. et al. Effects of microcurrent application and $670 \mathrm{~nm} \mathrm{InGaP} \mathrm{low-level} \mathrm{laser}$ irradiation on experimental wound healing in healthy and diabetic Wistar rats. Laser Physics, v. 23, n. 3, p. 035604-035607, 2013.

NORRED, C. L. Complementary and alternative medicine use by surgical patients. AORN J, v. 76, n. 6, p. 1013-1021, 2002.

PASSARINI JUNIOR, J. R. et al. Application of Jatropha curcas L. seed oil (Euphorbiaceae) and microcurrent on the healing of experimental wounds in Wistar rats. Acta Cirurgica Brasileira, v. 27, n. 7, p. 441-447, 2012. 
POLETTI, S. et. al. Electromagnetic Stimulation Combined with Aloe vera Increases Collagen Reorganization in Burn Repair. Journal of Pharmacy and Pharmacology, v. 6, n. 7, p. 633646, 2018.

RAO, S.G. et al. Atividade de cicatrização de feridas de Calendula officinalis e Hypericum: duas drogas homeopáticas que promovem a cicatrização de feridas em ratos. Fitoterapia, v. 62 , p. 508-10, 1991.

SPADARI, G. S. et al. Electrical stimulation enhances tissue reorganization during orthodontic tooth movement in rats. Clinical Oral Investigations, v. 21, n. 1, p. 111-120, 2016.

SÜNTAR, I. P. et al. Investigations on the in vivo wound healing potential of Hypericum perforatum L. Journal of Ethnopharmacology, v. 127, n. 2, p. 468-477, 2010.

TANGERINO FILHO, E. P. et al. Effects of microcurrent therapy on excisional elastic cartilage defects in young rats. Tissue and Cell, v. 48, n. 3, p.224-234, 2016.

UEMURA M., MAESHIGE N., KOGA Y., et al. Monophasic Pulsed 200- $\mu$ A Current Promotes Galvanotaxis With Polarization of Actin Filament and Integrin $\alpha 2 \beta 1$ in Human Dermal Fibroblasts. Eplasty, v. 16, n. 6, p. 325-332, 2016.

VANDENBOGAERDE, A., WITTE, P. Antineoplastic properties of photosensitized hypericin (Meeting). Anticancer Research, v.15, n.5A, p.1757-1758, 1995.

VEER, W.M.D. et al. Time course of the angiogenic response during normotrophic and hypertrophic scar formation in humans. Wound Repair and Regeneration, v. 19, n. 3, p. 292$301,2011$.

WANG, P.H. et al. Wound healing. Journal of the Chinese Medical Association, v. 81, n. 2, p. 94-101, 2018.

WILGUS, T. A. et al. Regulation of scar formation by vascular endothelial growth factor. Laboratory Investigation, v. 88, n. 6, p. 579-590, 21, 2008.

YU, C. et al. A novel microcurrent dressing for wound healing in a rat skin defect model. Military Medical Research, v. 6, n. 1, p. 327-345, 2019.

YU, C.; HU, Z.; PENG, R. Effects and mechanisms of a microcurrent dressing on skin wound healing: a review. Military Medical Research, v. 1, n. 1, p.24-27, 2014. 